\title{
Multidimensional prognostic indices for use in COPD patient care. A systematic review
}

\author{
Wouter D van Dijk ${ }^{*}$, Lisette van den Bemt ${ }^{1}$, Saskia van den Haak-Rongen ${ }^{2}$, Erik Bischoff ${ }^{1}$, Chris van Weel ${ }^{1}$, \\ Johannes CCM in 't Veen ${ }^{3}$ and Tjard RJ Schermer'
}

\begin{abstract}
Background: A growing number of prognostic indices for chronic obstructive pulmonary disease (COPD) is developed for clinical use. Our aim is to identify, summarize and compare all published prognostic COPD indices, and to discuss their performance, usefulness and implementation in daily practice.

Methods: We performed a systematic literature search in both Pubmed and Embase up to September 2010. Selection criteria included primary publications of indices developed for stable COPD patients, that predict future outcome by a multidimensional scoring system, developed for and validated with COPD patients only. Two reviewers independently assessed the index quality using a structured screening form for systematically scoring prognostic studies.

Results: Of 7,028 articles screened, 13 studies comprising 15 indices were included. Only 1 index had been explored for its application in daily practice. We observed 21 different predictors and 7 prognostic outcomes, the latter reflecting mortality, hospitalization and exacerbation. Consistent strong predictors were $\mathrm{FEV}_{1}$ percentage predicted, age and dyspnoea. The quality of the studies underlying the indices varied between fairly poor and good. Statistical methods to assess the predictive abilities of the indices were heterogenic. They generally revealed moderate to good discrimination, when measured. Limitations: We focused on prognostic indices for stable disease only and, inevitably, quality judgment was prone to subjectivity.
\end{abstract}

Conclusions: We identified 15 prognostic COPD indices. Although the prognostic performance of some of the indices has been validated, they all lack sufficient evidence for implementation. Whether or not the use of prognostic indices improves COPD disease management or patients' health is currently unknown; impact studies are required to establish this.

Keywords: COPD, index, multidimensional, prognosis

\section{Background}

Chronic obstructive pulmonary disease (COPD) is a chronic respiratory condition, with a high impact on patients' wellbeing, health care utilization, and mortality [1]. The (progressive) airflow obstruction that is characteristic for COPD is closely related to its morbidity and mortality [1-3]. Hence, degree of airflow obstruction is generally considered to be the key factor for staging COPD severity and to guide and monitor treatment [1]. However, (pharmacological) interventions to both

\footnotetext{
* Correspondence: w.vandijk@aios.umcn.nl

${ }^{1}$ Radboud University Nijmegen Medical Centre, Department of Primary and

Community Care, Nijmegen, the Netherlands

Full list of author information is available at the end of the article
}

stabilize the progression of airflow obstruction and reduce premature mortality are disappointing [4-6]. Currently, there is a growing recognition that COPD should no longer be regarded as a synonym for airflow obstruction alone, but instead as a multidimensional condition that comprises several phenotypes [1,7-10]. In addition, patients tend to die from other diseases than COPD [11]. Therefore, severity staging and treatment of COPD should follow this view and take into account the heterogenic nature of the disease.

Apart from airflow obstruction, a range of other factors have been identified as important predictors of future risk on morbidity and mortality in patients with COPD, including smoking [12,13], degree of dyspnoea [14], age

\section{C) Biomed Central}


[15], exercise capacity [16], body mass index [17], exacerbations [18], (cardiovascular) comorbidity [19], and quality of life [20]. Combining several of these prognostic factors in a multidimensional index embodies the current holistic vision on COPD, and may ultimately provide physicians with a powerful tool to assess and monitor disease severity in order to guide decision making and improve patient outcome [9,21]. Such an index clearly needs to meet several prerequisites in terms of accuracy, predictive and discriminative power, internal and external validity and, last but not least, practicability of use in different health care settings [22,23]. In addition to monitoring and guiding decision making in patients, a prognostic index could also be used to predict patients' health care utilization, to identify and target particular high-risk groups within the COPD patient population, or for risk stratification in clinical trials.

With the growing number of prognostic COPD indices developed for clinical use, an overview to facilitate the discussion of the pros and cons of using prognostic indices in COPD patient care is warranted. In this paper, we aim to identify, summarize and compare all published multidimensional prognostic indices that assess and stage disease severity in patients with COPD. Apart from providing insight in their performance, we aim to present the effects of their application in daily patient care, that will be important to help clinicians, scientists and health policy makers in deciding whether or not to implement an index.

\section{Methods}

We performed a systematic literature review on multidimensional prognostic indices for COPD.

\section{Literature search}

We comprehensively traced indices by systematically searching the Pubmed and Embase literature databases (see Additional File 1 and 2 for search strategies). We conducted the Pubmed search first and included all records published before 27 September 2010. The Pubmed search was not restricted to language. As the first index traced in Pubmed was published in 2004, the subsequent Embase search was limited to records published after 2002, and to records in English, German, French or Dutch. Two authors (LvdB and TS) independently screened the titles and abstracts of the retrieved records to identify potentially eligible studies, and next assessed eligibility by fulltext assessment. Disagreement was resolved by consensus, and if necessary by a third party $(\mathrm{SvdH})$. In addition, we searched the bibliographies of included articles for relevant cross-references and searched Pubmed and Embase for index-related articles reporting supplementary information on validity, usefulness, and/or additional prognostic outcomes.

\section{Study selection}

We included primary publications of prognostic indices that: 1$)$ were developed specifically for COPD; 2) were developed and validated in samples with COPD patients only; 3 ) were developed for assessment of COPD patients with stable disease (i.e., not during exacerbations or hospitalizations); 4) predict future outcome of COPD; 5) consist of more than one component; 6) have a scoring system for their components. As the quality of the prognostic indices was part of our evaluation we did not exclude indices based on poor quality.

\section{Data extraction and quality assessment}

The following data were extracted from each included study: age, gender, and airflow obstruction as measured by $\mathrm{FEV}_{1} / \mathrm{FVC}$ (forced expiratory volume in one second/forced vital capacity), $\mathrm{FEV}_{1} \%\left(\mathrm{FEV}_{1}\right.$ as percentage of the predicted value), and index purposes, predictors, prognostic outcomes and prognostic value. Ideally, a prognostic index progresses through three consecutive stages: development, validation, and impact quantification. There is no consensus on the 'best' method for development. Indices can be build from a set of predefined predictors that may either be established predictors and/or be selected based on practicability of measuring the predictor. The predictors could be selected based on their availability and pragmatism or by statistical modeling. The subsequent performance and validity of the developed index should be measured by its discriminative properties: how well does the index distinguish patients with poor prognosis from patients with good prognosis (c-statistic 0.50 equals random guessing, $>0.70$ is good performance, 1.0 is maximum), and by its accuracy (how well do predictions from the index measures up to the real observations, i.e. 'goodness-of-fit'). Since not all studies that report prognostic indices for COPD included this information in their methods, we additionally extracted data on correlation, multiple regression and survival analyses. To conclude the validation stage, an index should be investigated in a separate validation cohort in order to test generalization and, if applicable, it should be compared with conventional prognostic measures (in the case of COPD with $\mathrm{FEV}_{1} \%$. In general, if based on statistical selection of predictors and in smaller samples, the index is likely to perform better in its primary cohort. Before implementing an index in daily practice, the final challenge is to assess the effects on patient outcome and health care by impact studies [21-23].

$\mathrm{WvD}$ and LvdB independently scored the methodological quality of the studies using a structured screening form (Additional File 3), that exposes potential systematic bias and the validity of the generated indices: from good to poor, based on the average score of 6 items. This form was derived from a framework specifically developed for 
the evaluation of the methodological quality of prognosis studies in systematic reviews by Hayden et al, who extracted and grouped all quality items from 163 prognostic reviews [24].

\section{Results}

\section{Study selection}

Figure 1 summarizes the selection of studies. Of the 7,028 identified records, 31 were assessed for eligibility. After full-text assessment, we excluded 18 articles because of a lack of a scoring system for individual patients [25-27], absence of prognostic outcome [28-38], not being the original publication of an index [39-41], and not being COPD specific [42]. Consequently, we included 13 studies that altogether reported 15 multidimensional COPD indices (Table 1 and Additional Files 3 and 4). The first index was published in 2004 [43], whereas 2009 yields most studies. The prior purpose of most studies was to develop or improve an index for clinical use. Only 1 index was initially developed for epidemiologic use [30]. Index modeling was based on either achieving maximal predictive value or on practicability and mostly used statistical selection of predictors.

\section{Index descriptions}

The indices were established from study samples that ranged from 50 to 8802 patients, whereas their mean follow-up ranged from 0.5 to 6.1 years. The weighted average of $\mathrm{FEV}_{1} \%$ was $44 \%$ (study means range 35 to $63 \%)$. We observed large variation in predictors, scoring systems and outcomes (Table 1 and Additional File 4). From all indices, 21 predictors (Table 2) emerged for 7 different outcomes (Table 3). Across indices, shared predictors were often measured with different instruments, variously categorized, and diversely valued and weighted. For instance, 4 instruments were used to measure dyspnoea and 6 categorizations were applied to stage $\mathrm{FEV}_{1} \%$ (Additional File 4). Two of these categories deviated from the guidelines they referred to $[43,44]$, i.e. the cut-point of $65 \%$ was not mentioned in those guidelines $[45,46]$. Altogether, the indices included 3 to 8 predictors and their maximum scoring ranged from 8 to 422 points. Airflow obstruction - expressed as $\mathrm{FEV}_{1} \%$ was included in all indices except for one [47]. Other frequently included predictors were age, dyspnoea, exercise tolerance and Body Mass Index (BMI).

Mortality, as defined by all-cause or respiratory cause, was the most frequent predicted outcome $(n=8)$ $[15,43,44,48-51]$, followed by all-cause or respiratory hospitalization $(n=4)[47,48,52,53]$, exacerbation $(n=4)$ [48,52-54], and unscheduled in- and outpatient visits ( $\mathrm{n}=$ 1) [47]. Correlation with another index [43] was the only outcome in one study [55]. One study combined respiratory death and hospitalization as a composite outcome

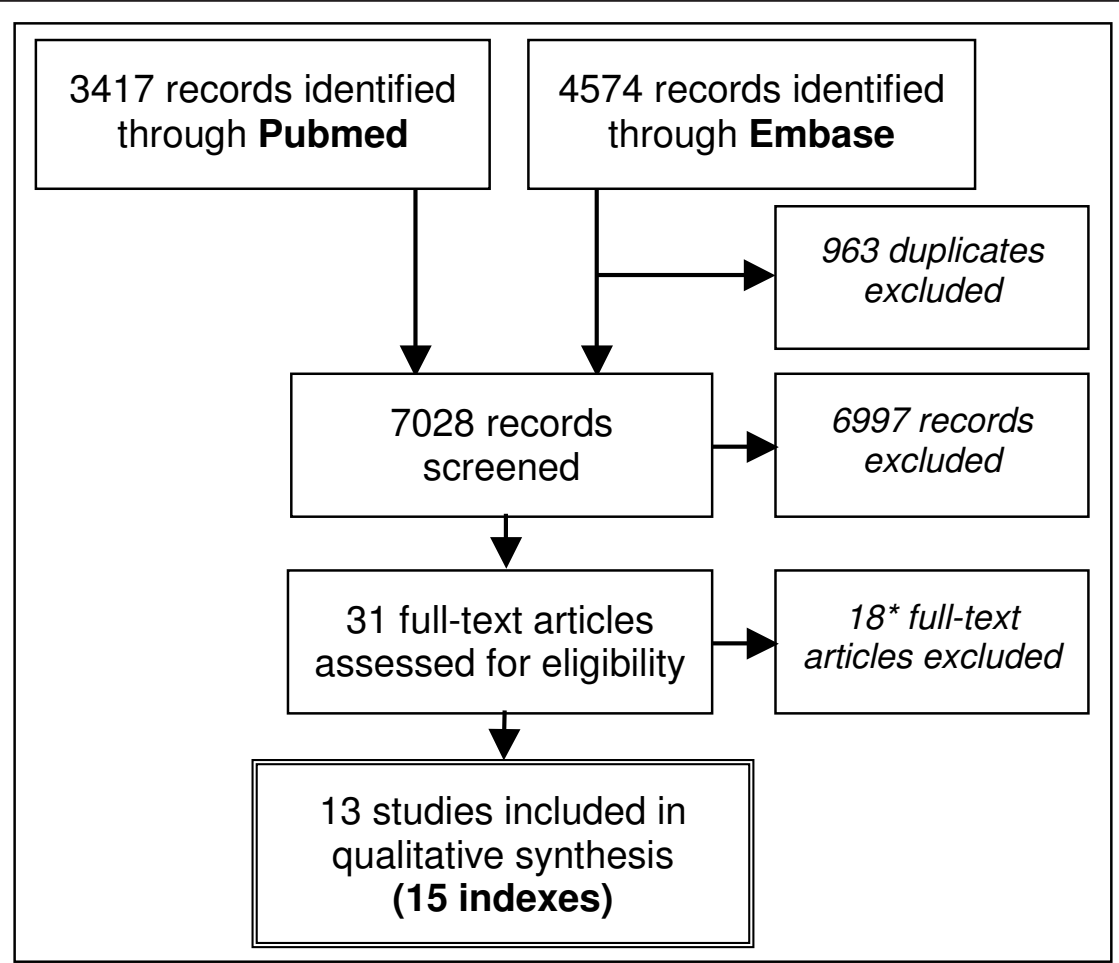

Figure 1 PRISMA Flow Diagram. * Three articles excluded based on lack of scoring system, 11 due to absence of prognostic outcome, 3 were not the original publication of the index, and 1 was not COPD specific. 
Table 1 Essential index summaries: general information, predictive ability, population and study quality.

\begin{tabular}{|c|c|c|c|c|c|c|c|c|c|c|c|c|c|c|c|}
\hline \multirow{2}{*}{$\begin{array}{l}\text { INDEX } \\
\text { Scale and } \\
\text { publication } \\
\text { year }\end{array}$} & \multicolumn{4}{|c|}{ GENERAL INDEX INFORMATION } & \multicolumn{4}{|c|}{$\underline{\text { INDEX QUALITY }}$} & \multicolumn{3}{|c|}{ POPULATION } & \multicolumn{2}{|c|}{$\underline{\text { STUDY QUALITY }}$} & \multirow[b]{2}{*}{$\begin{array}{l}\text { Val. } \\
\text { cohort }\end{array}$} & \multirow{2}{*}{$\begin{array}{l}\text { KEY STRENGTHS/ } \\
\text { FLAWS }\end{array}$} \\
\hline & $\begin{array}{l}\text { Index } \\
\text { aim }\end{array}$ & $\begin{array}{l}\text { Cited } \\
\text { (SCl) }\end{array}$ & Predictors & Outcome & $\begin{array}{l}\text { Predictor } \\
\text { ind. Sig. }\end{array}$ & $\begin{array}{l}\text { Discrimi } \\
\text { nation }\end{array}$ & $\begin{array}{l}\text { Accu } \\
\text { racy }\end{array}$ & $\begin{array}{l}\text { Compare } \\
\text { to }\end{array}$ & $N$ & $\begin{array}{l}\text { Age } \\
\text { (year) }\end{array}$ & $\begin{array}{l}\text { Mean } \\
\text { FEV }_{1} \%\end{array}$ & Score & $\begin{array}{l}\text { Model } \\
\text { building }\end{array}$ & & \\
\hline $\begin{array}{l}\text { ADO } \\
10 \text {-points scale } \\
2009\end{array}$ & $C$ & 14 & $\begin{array}{l}\text { Age } \\
\text { Dyspnoea } \\
\text { (MRC or GCRQ) } \\
\text { Obstruction } \\
(\mathrm{FEV}, \%)\end{array}$ & Death & Yes & Modest & Good & BODE & $\begin{array}{l}232 \\
342\end{array}$ & $\begin{array}{l}72 \\
68\end{array}$ & $\begin{array}{l}52 \% \\
\text { all }< \\
80 \%\end{array}$ & $\begin{array}{l}\text { Fairly } \\
\text { good }\end{array}$ & $A^{*}$ & + & $\begin{array}{l}\text { Accurate } \\
\text { Age is } \\
\text { paradoxical } \\
\text { Elderly patients } \\
\text { only }\end{array}$ \\
\hline $\begin{array}{l}\text { BODE } \\
\text { 10-point scale } \\
4 \text { categories } \\
2004\end{array}$ & C & 580 & $\begin{array}{l}\text { BMl (length/ } \\
\text { weight }^{2} \text { ) } \\
\text { Obstruction } \\
\text { (FEV } \% \text { ) } \\
\text { Dyspnoea } \\
\text { (MRC score) } \\
\text { Exercise } \\
\text { tolerance } \\
\text { (6MWD) }\end{array}$ & $\begin{array}{l}\text { Death } \\
\text { Respiratory death }\end{array}$ & Yes & $\begin{array}{l}\text { Death: } \\
\text { Good } \\
\text { Respiratory: } \\
\text { no report }\end{array}$ & - & $\mathrm{FEV}_{1} \%$ & $\begin{array}{l}207 \\
625\end{array}$ & $\begin{array}{l}66 \\
67\end{array}$ & $\begin{array}{l}39- \\
47 \%\end{array}$ & Good & $A^{*}$ & + & $\begin{array}{l}\text { Good quality } \\
\text { Good discrimination } \\
\text { Severe COPD only } \\
\text { Exclusion of CVD }\end{array}$ \\
\hline $\begin{array}{l}\text { BODEx } \\
\text { 9-point } \\
\text { scale } \\
4 \\
\text { categories } \\
2009\end{array}$ & $D$ & 7 & $\begin{array}{l}\text { BMI (length/ } \\
\text { weight }^{2} \text { ) } \\
\text { Obstruction } \\
\text { (FEV } \% \text { ) } \\
\text { Dyspnoea (MRC } \\
\text { score) } \\
\text { Exacerbations }\end{array}$ & Death & All but 1 & Good & - & $B O D E$ & 185 & 71 & $48 \%$ & Fair & $P$ & - & $\begin{array}{l}\text { Good discrimination } \\
\text { Elderly males } \\
\text { only }\end{array}$ \\
\hline $\begin{array}{l}\text { Qe-BODE } \\
\text { 12-point } \\
\text { scale } \\
4 \\
\text { categories } \\
2009\end{array}$ & $D$ & 7 & $\begin{array}{l}\text { Exacerbations } \\
\text { BMI (length/ } \\
\text { weight }^{2} \text { ) } \\
\text { Obstruction } \\
\text { (FEV } \% \text { ) } \\
\text { Dyspnoea (MRC } \\
\text { score) } \\
\text { Exercise } \\
\text { tolerance } \\
\text { (6MWD) }\end{array}$ & Death & All but 1 & Good & - & $B O D E$ & 185 & 71 & $48 \%$ & Fair & A & - & $\begin{array}{l}\text { Good discrimination } \\
\text { Elderly males } \\
\text { only }\end{array}$ \\
\hline $\begin{array}{l}\triangle m B O D E \\
\text { 10-point } \\
\text { scale } \\
2007\end{array}$ & $D$ & 3 & $\begin{array}{l}\text { BMI (length/ } \\
\text { weight }{ }^{2} \text { ) } \\
\text { Obstruction } \\
\left.\text { (FEV }{ }^{\prime} \%\right) \\
\text { Dyspnoea (MRC } \\
\text { score) } \\
\text { Exercise max. } \\
\mathrm{O}_{2} \text {-use }\end{array}$ & Correlation BODE & & - & - & $B O D E$ & 50 & 63 & $63 \%$ & Fair & $A$ & - & $\begin{array}{l}\text { Small sample } \\
\text { Restricted } \\
\text { outcome and } \\
\text { analysis }\end{array}$ \\
\hline
\end{tabular}


Table 1 Essential index summaries: general information, predictive ability, population and study quality. (Continued)

\begin{tabular}{|c|c|c|c|c|c|c|c|c|c|c|c|c|c|c|c|}
\hline $\begin{array}{l}\text { COPDSS: } \\
\text { COPD } \\
\text { Severity } \\
\text { Score } \\
\text { 35-point scale } \\
2008\end{array}$ & $E$ & 7 & $\begin{array}{l}\text { Respiratory } \\
\text { symptoms } \\
\text { Systemic } \\
\text { corticosteroids } \\
\text { Other COPD } \\
\text { medications } \\
\text { Hospitalization/ } \\
\text { Intubation } \\
\text { Home oxygen } \\
\text { use }\end{array}$ & $\begin{array}{l}\text { Respiratory outpatient } \\
\text { Respiratory ED visit } \\
\text { Respiratory hospital }\end{array}$ & $\begin{array}{l}\text { - } \\
\text { (index } \\
\text { yes) }\end{array}$ & - & - & - & 267 & 65 & $54 \%$ & Fair & $P$ & $\pm^{\$}$ & $\begin{array}{l}\text { Nomogram } \\
\text { Diagnosis based } \\
\text { on self-report } \\
\text { No outcome } \\
\text { confirmation }\end{array}$ \\
\hline $\begin{array}{l}\text { CPI: COPD } \\
\text { Prognostic } \\
\text { Index } \\
100 \text {-point } \\
\text { scale } \\
3 \text { categories } \\
2008\end{array}$ & C & 2 & $\begin{array}{l}\text { Quality of life } \\
\text { (SGRQ/CRQ) } \\
\text { Obstruction } \\
\text { (FEV } \% \text { ) } \\
\text { Age } \\
\text { Gender } \\
\text { BMl } \\
\text { History of ED/ } \\
\text { exacerbation } \\
\text { History of CVD }\end{array}$ & $\begin{array}{l}\text { Death } \\
\text { Hospitalization } \\
\text { Exacerbation }\end{array}$ & $\begin{array}{l}\text { Depends } \\
\text { on } \\
\text { outcome }\end{array}$ & $\begin{array}{l}\text { Model: } \\
\text { Good } \\
\text { Val.cohort: } \\
\text { no report }\end{array}$ & - & - & $\begin{array}{l}5856 \\
2946\end{array}$ & $\begin{array}{l}64 \\
64\end{array}$ & $44 \%$ & $\begin{array}{l}\text { Fairly } \\
\text { poor }\end{array}$ & $P^{*}$ & + & $\begin{array}{l}\text { Adequate statistics } \\
\text { Large sample } \\
\text { Selective } \\
\text { reporting } \\
\text { Pooled analysis }\end{array}$ \\
\hline $\begin{array}{l}\text { DOREMI BOX } \\
10 \text {-point scale } \\
2 \text { categories } \\
2008\end{array}$ & D & $?$ & $\begin{array}{l}\text { Dyspnoea } \\
\text { (ATS) } \\
\text { Obstruction } \\
\left.\text { (FEV }{ }_{1} \%\right) \\
\text { Rate of } \\
\text { Exacerbation } \\
\text { Movement } \\
\text { (6MWD) } \\
\text { BMI (length/ } \\
\left.\text { weight }{ }^{2}\right) \\
\mathrm{Blood} \mathrm{OXygen}_{\left(\mathrm{PaO}_{2}\right)}\end{array}$ & $\begin{array}{l}\text { Correlation BODE } \\
\text { Death }\end{array}$ & $\begin{array}{l}\text { Yes } \\
\text { (2 no } \\
\text { report) }\end{array}$ & - & - & BODE & 84 & 59 & $\begin{array}{l}35 \% \\
(18- \\
73 \%)\end{array}$ & $\begin{array}{l}\text { Fairly } \\
\text { good }\end{array}$ & A & - & $\begin{array}{l}\text { Clear descriptions } \\
\text { Long follow-up } \\
\text { Small sample } \\
\text { Limited statistics } \\
\text { Severe } \\
\text { population }\end{array}$ \\
\hline $\begin{array}{l}\text { DOSE, } \\
\text { 8-point scale } \\
2009\end{array}$ & C & 3 & $\begin{array}{l}\text { Dyspnoea } \\
\text { (MRC score) } \\
\text { Obstruction } \\
\text { (FEV } \% \text { ) } \\
\text { Smoking } \\
\text { Exacerbations }\end{array}$ & $\begin{array}{l}\text { Correlation BODE } \\
\text { Exacerbation } \\
\text { Hospitalization for } \\
\text { exacerbation }\end{array}$ & $\begin{array}{l}\text { - } \\
\text { (index } \\
\text { partly) }\end{array}$ & $\begin{array}{l}\text { Hospital } \\
\text { Good } \\
\text { Exacerbation } \\
\text { no report }\end{array}$ & - & BODE & $\begin{array}{l}375 \\
81 \\
133\end{array}$ & $\begin{array}{l}69 \\
73 \\
67\end{array}$ & $\begin{array}{l}42- \\
67 \% \\
\text { all }< \\
80 \%\end{array}$ & $\begin{array}{l}\text { Fairly } \\
\text { poor }\end{array}$ & $\mathrm{P}^{*}$ & $t^{\#}$ & $\begin{array}{l}\text { Difficult, complex } \\
\text { and selective } \\
\text { reporting } \\
\text { Violated own } \\
\text { protocol }\end{array}$ \\
\hline $\begin{array}{l}\text { HADO } \\
\text { 12-point scale } \\
3 \text { categories } \\
2006\end{array}$ & C & 5 & $\begin{array}{l}\text { Health (new } \\
\text { questionnaire) } \\
\text { Activity (new } \\
\text { questionnaire) } \\
\text { Dyspnoea } \\
\text { (Fletcher) } \\
\text { Obstruction } \\
\text { (FEV } \% \text { ) }\end{array}$ & Death & No & Modest & - & $\mathrm{FEV}_{1} \%$ & 611 & 67 & $\begin{array}{l}50 \% \\
\text { all }< \\
80 \%\end{array}$ & $\begin{array}{l}\text { Fairly } \\
\text { good }\end{array}$ & $P$ & - & $\begin{array}{l}\text { Clear descriptions } \\
\text { Compared to } \mathrm{FEV}_{1} \% \\
\text { Modest } \\
\text { discrimination } \\
\text { Predictors } \\
\text { debatable }\end{array}$ \\
\hline
\end{tabular}


Table 1 Essential index summaries: general information, predictive ability, population and study quality. (Continued)

\begin{tabular}{|c|c|c|c|c|c|c|c|c|c|c|c|c|c|c|c|}
\hline $\begin{array}{l}\text { Niewoehner } \\
\text { (1) } \\
\text { 422-point } \\
\text { scale } \\
2007\end{array}$ & $C$ & 27 & $\begin{array}{l}\text { Age } \\
\text { Obstruction } \\
\text { (FEV } \% \text { ) } \\
\text { Hospitalization } \\
\text { COPD duration } \\
\text { Productive } \\
\text { cough } \\
\text { Antibiotics } \\
\text { Systemic } \\
\text { corticosteroids } \\
\text { Theophylline }\end{array}$ & Exacerbation & Yes & Modest & $\begin{array}{l}\text { Seems } \\
\text { good }^{@}\end{array}$ & - & 1829 & 69 & $\begin{array}{l}36 \% \\
\text { all< } \\
60 \%\end{array}$ & Fair & $A^{*}$ & - & $\begin{array}{l}\text { Large sample } \\
\text { No validation } \\
\text { cohort } \\
\text { Severe COPD/ } \\
\text { males only } \\
\text { No outcome } \\
\text { confirmation }\end{array}$ \\
\hline $\begin{array}{l}\text { Niewoehner } \\
\text { (2) } \\
\text { 249-point } \\
\text { scale } \\
2007\end{array}$ & $C$ & 27 & $\begin{array}{l}\text { Age } \\
\text { Obstruction } \\
\left(\text { FEV }{ }_{1} \%\right) \\
\text { Hospitalization } \\
\text { Unscheduled } \\
\text { visits } \\
\text { Cardiovascular } \\
\text { disease } \\
\text { Oral } \\
\text { corticosteroids }\end{array}$ & $\begin{array}{l}\text { Hospitalization for } \\
\text { exacerbation }\end{array}$ & Yes & Good & $\begin{array}{l}\text { Seems } \\
\text { good }^{@}\end{array}$ & - & 1829 & 69 & $\begin{array}{l}36 \% \\
\text { all }< \\
60 \%\end{array}$ & Fair & $A^{*}$ & - & $\begin{array}{l}\text { Good discrimination } \\
\text { Large sample } \\
\text { No validation } \\
\text { cohort } \\
\text { Severe COPD/ } \\
\text { males only } \\
\text { No outcome } \\
\text { confirmation } \\
\text { Predictor is } \\
\text { outcome }\end{array}$ \\
\hline $\begin{array}{l}\text { PILE } 2010 \\
\text { 10-point scale } \\
4 \text { categories }\end{array}$ & C & 0 & $\begin{array}{l}\text { Obstruction } \\
\left(\mathrm{FEV}_{1} \%\right) \\
\text { Interleukin-6 } \\
\text { Knee extensor } \\
\text { strength }\end{array}$ & Death & Yes & Good & - & $\begin{array}{l}\mathrm{FEV}_{1} \% \\
\mathrm{mBODE}\end{array}$ & 268 & 73 & $\begin{array}{l}63 \% \\
\text { all > } \\
30 \%\end{array}$ & Fair & $A^{*}$ & - & $\begin{array}{l}\text { Long follow-up } \\
\text { Good statistics } \\
\text { No validation } \\
\text { cohort }\end{array}$ \\
\hline $\begin{array}{l}\text { SAFE } 2007 \\
9 \text {-point scale } \\
4 \text { categories }\end{array}$ & C & 5 & $\begin{array}{l}\text { SGRQ score } \\
\text { (questionnaire) } \\
\text { Air-flow } \\
\text { limitation } \\
\text { (FEV } \% \text { ) } \\
\text { Exercise } \\
\text { tolerance } \\
\text { (6MWD) }\end{array}$ & $\begin{array}{l}\text { Exacerbation } \\
\text { (correlation) }\end{array}$ & - & - & - & - & 86 & 68 & $\begin{array}{l}43 \% \\
(12- \\
98 \%)\end{array}$ & Fair & $P$ & - & $\begin{array}{l}\text { Small sample } \\
\text { Poor statistics }\end{array}$ \\
\hline $\begin{array}{l}\text { Schembri } \\
\text { (TARDIS) } \\
16 \text {-point scale } \\
2009\end{array}$ & $C$ & 0 & $\begin{array}{l}\text { Age } \\
\text { BMl } \\
\text { Dyspnoea } \\
\text { (MRC score) } \\
\text { Obstruction } \\
\text { (FEV }{ }_{1} \% \text { ) } \\
\text { Hospitalization } \\
\text { Influenza } \\
\text { vaccination }\end{array}$ & $\begin{array}{l}\text { Hospitalization for COPD } \\
\text { or respiratory death as } 1 \\
\text { outcome }\end{array}$ & Yes & - & - & - & 3343 & $?$ & $\begin{array}{l}? \\
\text { all }< \\
80 \%\end{array}$ & $\begin{array}{l}\text { Fair to } \\
\text { fairly } \\
\text { poor }\end{array}$ & $A^{*}$ & - & $\begin{array}{l}\text { Large sample } \\
\text { No validation } \\
\text { cohort } \\
\text { Composite } \\
\text { outcome } \\
\text { Limited statistics }\end{array}$ \\
\hline
\end{tabular}

Indices are sorted alphabetically. Index aim: primary purpose of utilization: C) clinical use, D) further development of existing index, E) epidemiologic use; Score: average of bias screening form; Model building: priorities in model development (* if predictors are statistically selected): A) accuracy, P) pragmatism; Val. cohort: separate validation cohort; SCl: science citation index; Predictor ind. sig.: independent significance of predictors for outcome; \$: same cohort, different time-window; \#: Selective use and reporting of cohorts; $;$ : reliability plot without statistics. CVD: cardiovascular disease 
Table 2 COPD outcome predictors $(n=21)$, grouped by disease components

\begin{tabular}{|c|c|c|}
\hline COMPONENT & PREDICTOR & $\mathrm{N}$ \\
\hline \multirow[t]{3}{*}{ Physiologic } & - Obstruction (FEV, as \% from predicted) & 14 \\
\hline & - Blood oxygen ( $\mathrm{PaO} 2)$ & 1 \\
\hline & - Exercise maximum oxygen consumption & 1 \\
\hline Biomarkers & - Interleukin-6 & 1 \\
\hline \multirow[t]{3}{*}{ Physical } & - BMI (length/weight2) & 7 \\
\hline & - Exercise tolerance (6MWD) & 4 \\
\hline & - Knee extensor strength & 1 \\
\hline \multirow[t]{3}{*}{ Medical history } & - COPD duration & 1 \\
\hline & - (Rate of) exacerbation (unscheduled visits, emergency department visits, hospitalisation, intubation) & 8 \\
\hline & - History of cardiovascular disease & 2 \\
\hline \multirow[t]{4}{*}{ Demographics } & - Age & 5 \\
\hline & - Gender & 1 \\
\hline & - Smoking status & 1 \\
\hline & - Influenza vaccination status & 1 \\
\hline \multirow[t]{4}{*}{ Signs \& symptoms } & - Dyspnoea (ATS, MRC score, GCRQ, and Fletcher) & 9 \\
\hline & - Quality of life (SGRQ) & 2 \\
\hline & - Health status (CRQ and ad hoc questionnaires) & 2 \\
\hline & - Respiratory symptoms like cough & 2 \\
\hline \multirow[t]{3}{*}{ Treatment } & - COPD medication (including steroids and theophylline) & 3 \\
\hline & - Antibiotics & 1 \\
\hline & - Home oxygen & 1 \\
\hline
\end{tabular}

$\mathrm{N}$ is number of indices.

[56]. Two studies also analyzed the change of the index over time and the association with outcome [47,52]. Definitions and methods of measurement of shared outcomes varied across indices, particularly for exacerbations (Additional File 3).

\section{Performance and quality}

Table 1 reveals the statistical methods used to validate the performance of the respective indices, which were heterogenic and often deficient. Only 3 indices were compared to $\mathrm{FEV}_{1} \%$ and showed a modest, not formally tested, improvement of discriminative power in contrast to $\mathrm{FEV}_{1} \%$ (c-statistics increased from $0.63-0.65$ to 0.68 - 0.74). Only 5 studies used some sort of validation

Table 3 Summary of different outcomes used in prognostic COPD indexes

\begin{tabular}{lll}
\hline OUTCOME & SPECIFIC INDEX OUTCOME & N \\
\hline Death & - All-cause death & 8 \\
\multirow{3}{*}{ Hospitalisation } & - Respiratory death & $2^{*}$ \\
& - All-cause hospitalization & 1 \\
Exacerbation & - Respiratory hospitalization & $4^{*}$ \\
& - Unscheduled respiratory outpatient visit & 1 \\
& - Respiratory emergency department visit & 1 \\
& - Any exacerbation & 4 \\
\hline
\end{tabular}

$\mathrm{N}$ is number of studies. ${ }^{*}$ One study used these outcomes as a composite outcome cohort. Apart from these primary studies, only 2 indices had been additionally validated for their outcomes in succeeding studies $[43,55]$, whereas 3 indices had been additionally evaluated for other outcomes (Additional File 4) $[43,48,55]$. Remarkably, we retrieved only 1 (noncontrolled pilot) impact study that implemented a multidimensional prognostic index in COPD patient care (11 patients included, 6 months follow-up, no significant improvements due to case management directed by the index) [57].

The overall methodological quality varied from fairly poor to good (Table 1). All studies described the baseline characteristics of their sample adequately, but only half described their process of patient recruitment and selection. Nine studies did not properly report the study attrition, information on drop-outs in particular, and one study appeared to violate its own protocol for predictor selection [52]. Measurements sometimes remained undefined and were inadequate for several indices, in particular with respect to (lack of) outcome confirmation.

\section{Discussion}

We identified 15 different multidimensional prognostic COPD indices, of which several have been validated appropriately. Although most indices were developed for clinical use, as yet, these indices lack impact studies to demonstrate effects on patient outcome and health care 
when implemented in daily patient care. The indices may improve population-based prediction of the natural course of COPD compared to looking at airway obstruction (as measured by $\mathrm{FEV}_{1} \%$ ) alone, in terms of mortality, hospitalizations and exacerbations, although discrimination still remains modest. The diversity in populations, (the weighting of) predictors and (the definition of) outcome, hampers any overall recommendations on which index to prefer for predicting prognosis in patients with COPD. However, our overview of the indices currently available can guide future research in selecting the most suitable index/indices for impact studies.

\section{Strengths and limitations}

Our systematic search in two leading medical literature databases limited the chance of missing an index. The Embase search did not reveal additional indices. As there is no consensus on how to perform quality assessment of studies in systematic reviews of prognostic studies, we based our study assessments on Hayden's previously reported criteria, in an effort to enhance (the validity of) our study evaluations [24]. A limitation of our review is the (inevitable) subjectivity when judging the methodological quality of the studies underlying the indices, including deficient blinding of the studies. We attempted to counteract subjectivity by independent and systematic scoring by two investigators. Finally, we focused on prognostic indices for stable disease only and excluded indices that had been developed for acute exacerbations, or that assessed current disease severity only without predicting future outcome(s).

\section{Interpretation}

Although the quality of the studies generally appeared fair, the lack of proper analyses on their predictive performance and the lack of uniformity across indices hampers any recommendations regarding preference for any particular index. Altogether, rather than truly improving predictive abilities, the large number of COPD indices mainly substantiate the important value of airflow obstruction on population level. Although regarded as a relatively difficult and impractical measurement to obtain, this single predictor is required for the diagnosis of COPD [1]. Most other predictors would be relatively easy to perform, except for time and space consuming exercise tests, of which age and dyspnoea in particular seem reliable and predictive measures.

As smoking is an important, easily measured and modifiable predictor of COPD prognosis, we were surprised to observe that of 6 studies that reported smoking, only 1 (fairly poor) study included smoking in its index. Seven studies did not have information about smoking available. Obviously, smoking status and smoking intensity may change repeatedly over time and self-reported reliability is poor, requiring confirmation tests like urine analyses. Furthermore, age is an excellent predictor in both cardiovascular and COPD indices [58]. However, age seems a difficult predictor to value in a prognostic index for secondary prevention, such as with COPD [59]. Age is non-modifiable and its usefulness in tailoring individual COPD treatment accordingly is less obvious: it may prohibit treatment of young patients, and younger patients would ironically have less urgency to quit smoking.

Although the COPD indices could be used for epidemiologic and research purposes, they would ideally support (cost-effective) decision making on individual treatment in order to improve outcome in patients with COPD [9,21], or even as to provide individual prognoses. So far, only one (pilot) study actually implemented a prognostic COPD index in patient care, without showing significant improvements in health (care) [57]. As the practical abilities of COPD indices remain unexposed, they fail to tackle the current urge to improve treatment programs $[7,9,60]$.

Ongoing research by prospective follow-up of a wide range of (known and new) predictors specifically aims to further improve both phenotyping and the prognostic capacity of COPD indices [61,62]. However, as the 'proof of the pudding' would be to actually study the effect of applying such an index in directing decision making and outcome in individual patients, research priorities would need to shift towards these objectives, preferably by means of impact studies $[9,21,23]$. Different indices might prove to be suitable for different populations, purposes and settings [63]. Although impact studies have not (yet) been used commonly for prognostic indices [23], we believe that COPD treatments with variable effects further urge to study the effects of implementing an index in daily practice.

\section{Recommendations}

Following the development and validation of the current indices, the next step for COPD researchers is to perform impact studies, instead of developing more indices [23]. These studies should establish both the applicability and the impact on health(care) of implementation of these indices in daily clinical practice. An impact study requires a randomized controlled trial that quantifies the effect of using a prognostic index on predefined outcomes including decision making, patient outcome, and cost effectiveness, compared with usual care without implementing the index [23].

Another issue is whether or not a prognostic index should integrate predictors, preferably modifiable [9], of which treatments can indeed improve the index scores and outcome $[64,65]$. Ultimately, indices should be integrated with predictive, preventive, personalized and participatory 
(P4) medicine [66], i.e. index-tailored (self)management strategies for individual COPD phenotypes. To improve applicability in clinical practice, a $5^{\text {th }} \mathrm{P}$ could be added for 'practical'.

Finally, applying a prognostic index in a patient population other than the one in which it was developed may require recalibration or modification. For example, Puhan et al. recently reported improved prognostic performance of the BODE index after population-based recalibration [15]. Age-specific calibration or stratification of an index allows adjustments that can resolve the dilemma of this strong but troublesome predictor [58]. Apart from age, calibration to critically-ill patients in need of (decisions on their) ICU admission and/or ventilation, might prove its benefit. In addition, this population could also benefit from non-modifiable predictors that merely define a highrisk group of patients [67].

\section{Conclusions}

We identified 15 multidimensional prognostic indices specifically developed for COPD patients. Although the overall prognostic performance appears moderate to good and several indices have been validated, there currently is a lack of evidence that prognostic indices improve decision making, treatment or outcome in patients with COPD. The next challenge is to perform impact studies that determine if implementation of specific indices can indeed improve individual health(care) in specific settings.

\section{Additional material}

Additional file 1: Pubmed Database Search: Pubmed Search strategy. Additional file 2: Embase Database Search: Embase Search strategy. Additional file 3: Index Quality Assessments: Contains the quality assessment form and all index quality assessments.

Additional file 4: Detailed Index Summaries: Contains a more extensive summary of the index characteristics and properties.

\section{Acknowledgements}

The principal investigator ( $W v D$ ) had full access to all of the data in the study and takes responsibility for the integrity of the data and the accuracy of the data analysis.

Funding: The paper was supported by a university grant (RvB08.066.51196/ GE) by Radboud University Nijmegen Medical Center, Nijmegen, the Netherlands, which has no interference with the study with regards to design, data collection, analyses, manuscript submission or whatsoever. We wish to thank Ms. Elmie Peters for her advice in the literature search.

\section{Author details}

${ }^{1}$ Radboud University Nijmegen Medical Centre, Department of Primary and Community Care, Nijmegen, the Netherlands. ${ }^{2}$ QUARTZ Integrated Care Support Service, Helmond, the Netherlands. ${ }^{3}$ Sint Franciscus Gasthuis, Department of Chest Diseases, Rotterdam, the Netherlands.

\section{Authors' contributions}

All authors read and approved the final manuscript. WvD: Acquisition and interpretation of data, drafting of the manuscript. LvdB: Study concept and design, acquisition and interpretation of data, drafting of the manuscript. SvdH: Drafting of the manuscript. EB: Drafting of the manuscript. CvW: Drafting and critical revision of the manuscript. JiV: Drafting and critical revision of the manuscript. TS: Study concept, design and supervision, acquisition and interpretation of data, drafting and critical revision of the manuscript

\section{Competing interests}

There are no conflicts of interest of any kind related to this paper. Conflicts of interest, not related to this paper: Johannes in 't Veen received fees for consultancy or lectures from Astra Zeneca, Boehringer Ingelheim, Cheisi, Novartis and Nycomed. Tjard Schermer received fees or grants for his department used for research, education, equipment, salaries, etc. from NutsOhra Fund, GlaxoSmithKline, AstraZeneca and Boehringer Ingelheim. Chris van Weel received unrestricted fees or grants for his department for research, education, equipment, salaries, etc. from Bayer, NovoNordisk, Astra Zeneca, Boehringer Ingelheim, GlaxoSmithKline and Novartis. Wouter van Dijk, Lisette van den Bemt, Erik Bischoff and Saskia van den Haak-Rongen do not have any conflict of interest.

Received: 20 April 2011 Accepted: 14 November 2011 Published: 14 November 2011

\section{References}

1. Global Initiative for Chronic Obstructive Lung Disease (GOLD): Global Strategy for Diagnosis, Management, and Prevention of COPD (2010). [http://www.goldcopd.com].

2. Mannino DM, Reichert MM, Davis KJ: Lung function decline and outcomes in an adult population. Am J Respir Crit Care Med 2006, 173(9):985-990.

3. Anthonisen NR, Wright EC, Hodgkin JE, IPPB Trial Group: Prognosis in chronic obstructive pulmonary disease. Am Rev Respir Dis 1986, 133:14-20.

4. Tashkin DP, Celli B, Senn S, Burkhart D, Kesten S, Menjoge S, Decramer M: A 4-year trial of tiotropium in chronic obstructive pulmonary disease. $\mathrm{N}$ Engl J Med 2008, 359(15):1543-1554.

5. Yang IA, Fong KM, Sim EH, Black PN, Lasserson TJ: Inhaled corticosteroids for stable chronic obstructive pulmonary disease. Cochrane Database Syst Rev 2007, 2: CD002991.

6. Calverley PM, Anderson JA, Celli B, Ferguson GT, Jenkins C, Jones PW, Yates JC, Vestbo J: Salmeterol and fluticasone propionate and survival in chronic obstructive pulmonary disease. N Engl J Med 2007, 356(8):775-789.

7. van den Bemt L, Schermer TRJ: Multicomponent staging indices for COPD in daily patient care: what's the yield? Int J Clin Pract 2010, 64(11):1475-1479.

8. Mannino DM, Buist AS: Global burden of COPD: risk factors, prevalence, and future trends. Lancet 2007, 370(9589):765-773.

9. Schunemann $\mathrm{H}$ : From BODE to ADO to outcomes in multimorbid COPD patients. Lancet 2009, 374(9691):667-668.

10. Han MK, Agusti A, Calverley PM, Celli BR, Criner G, Curtis JL, Fabbri LM, Goldin JG, Jones PW, MacNee W, Make BJ, Rabe KF, Rennard SI, Sciurba FC, Silverman EK, Vestbo J, Washko GR, Wouters EF, Martinez FJ: Chronic obstructive pulmonary disease phenotypes: the future of COPD. Am J Respir Crit Care Med 2010, 182(5):598-604.

11. Berry CE, Wise RA: Mortality in COPD: causes, risk factors, and prevention. COPD 2010, 7(5):375-382.

12. Godtfredsen NS, Lam TH, Hansel T, Leon ME, Gray N, Dresler C, Burns DM, Prescott E, Vestbo J: COPD-related morbidity and mortality after smoking cessation: status of the evidence. Eur Respir J 2008, 32(4):844-853.

13. Ezzati M, Lopez AD: Estimates of global mortality attributable to smoking in 2000. Lancet 2003, 362(9387):847-852.

14. Nishimura K, Izumi T, Tsukino M, Oga T: Dyspnea is a better predictor of 5 -year survival than airway obstruction in patients with COPD. Chest 2002, 121(5):1434-1440.

15. Puhan MA, Garcia-Aymerich J, Frey M, ter Riet G, Anto JM, Agusti AG, Gomez FP, Rodriguez-Roisin R, Moons KG, Kessels AG, Held U: Expansion of the prognostic assessment of patients with chronic obstructive pulmonary disease: the updated BODE index and the ADO index. Lancet 2009, 374(9691):704-711.

16. Oga T, Nishimura $K$, Tsukino M, Sato $S$, Hajiro $T$ : Analysis of the factors related to mortality in chronic obstructive pulmonary disease: role of exercise capacity and health status. Am J Respir Crit Care Med 2003, 167(4):544-549. 
17. Landbo C, Prescott E, Lange P, Vestbo J, Almdal TP: Prognostic value of nutritional status in chronic obstructive pulmonary disease. Am J Respir Crit Care Med 1999, 160(6):1856-1861.

18. Donaldson GC, Wedzicha JA: COPD exacerbations 1: Epidemiology. Thorax 2006, 61(2):164-168.

19. Mannino DM, Thorn D, Swensen A, Holguin F: Prevalence and outcomes of diabetes, hypertension and cardiovascular disease in COPD. Eur Respir J 2008, 32(4):962-969.

20. Fan VS, Curtis JR, Tu SP, McDonell MB, Finn SD: Using quality of life to predict hospitalization and mortality in patients with obstructive lung diseases. Chest 2002, 122(2):429-436.

21. Moons KG, Royston P, Vergouwe Y, Grobbee DE, Altman DG: Prognosis and prognostic research: what why and how? BMJ 2009, 338:b375.

22. Altman DG, Vergouwe $Y$, Royston P, Moons KG: Prognosis and prognostic research: validating a prognostic model. BMJ 2009, 338:b605.

23. Moons KG, Altman DG, Vergouwe $Y$, Royston P: Prognosis and prognostic research: application and impact of prognostic models in clinical practice. BMJ 2009, 338:b606.

24. Hayden JA, Cote P, Bombardier C: Evaluation of the quality of prognosis studies in systematic reviews. Ann Intern Med 2006, 144(6):427-437.

25. Fan VS, Ramsey SD, Make BJ, Martinez FJ: Physiologic variables and functional status independently predict COPD hospitalizations and emergency department visits in patients with severe COPD. COPD 2007 4(1):29-39.

26. Atis S, Kanik A, Ozgur ES, Eker S, Tumkaya M, Ozge C: [How exactly can we predict the prognosis of COPD]. Tuberk Toraks 2009, 57(3):289-297.

27. Burgel PR, Paillasseur JL, Caillaud D, Tillie-Leblond I, Chanez P, Escamilla R, Court-Fortune Perez T, Carre P, Roche N: Clinical COPD phenotypes: a novel approach using principal component and cluster analyses. Eur Respir J 2010, 36(3):472-4

28. Bailey WC, Sciurba FC, Hanania NA, Donohue JF, Ferguson GT, Zibrak JD, Sharafkhaneh A, Marcus P, Rosa K, Piault EC, Martinez FJ: Development and validation of the Chronic Obstructive Pulmonary Disease Assessment Questionnaire (COPD-AQ). Prim Care Respir J 2009, 18(3):198-207.

29. Celli BR, Calverley PM, Rennard SI, Wouters EF, Agusti A, Anthonisen N, MacNee W, Jones P, Pride N, Rodriguez-Roisin R, Rossi A, Wanner A: Proposal for a multidimensional staging system for chronic obstructive pulmonary disease. Respir Med 2005, 99(12):1546-1554.

30. Eisner MD, Trupin L, Katz PP, Yelin EH, Earnest G, Balmes J, Blanc PD: Development and validation of a survey-based COPD severity score. Chest 2005, 127(6):1890-1897.

31. Jones P, Harding G, Wiklund I, Berry P, Leidy N: Improving the process and outcome of care in COPD: development of a standardised assessment tool. Prim Care Respir J 2009, 18(3):208-215.

32. Miravitlles M, Llor C, de Castellar R, Izquierdo I, Baro E, Donado E: Validation of the COPD severity score for use in primary care: the NEREA study. Eur Respir J 2009, 33(3):519-527.

33. Peters JB, Daudey L, Heijdra YF, Molema J, Dekhuijzen PN, Vercoulen JH: Development of a battery of instruments for detailed measurement of health status in patients with COPD in routine care: the Nijmegen Clinical Screening Instrument. Qual Life Res 2009, 18(7):901-912.

34. Selim AJ, Ren XS, Fincke G, Rogers W, Lee A, Kazis LA: Symptom-based measure of the severity of chronic lung disease: results from the Veterans Health Study. Chest 1997, 111(6):1607-1614.

35. Vercoulen JH, Daudey L, Molema J, Vos PJ, Peters JB, Top M, Folgering H: An Integral assessment framework of health status in chronic obstructive pulmonary disease (COPD). Int J Behav Med 2008, 15(4):263-279

36. Verhage TL, Heijdra YF, Molema J, Daudey L, Dekhuijzen PN, Vercoulen JH: Adequate Patient Characterization in COPD: Reasons to Go Beyond GOLD Classification. Open Respir Med J 2009, 3:1-9.

37. Wu EQ, Birnbaum HG, Cifaldi M, Kang Y, Mallet D, Colice G: Development of a COPD severity score. Curr Med Res Opin 2006, 22(9):1679-1687.

38. Yeh ML, Chen HH, Liao YC, Liao WY: Testing the functional status model in patients with chronic obstructive pulmonary disease. J Adv Nurs 2004, 48(4):342-350

39. Cote CG, Celli BR: BODE index: a new tool to stage and monitor progression of chronic obstructive pulmonary disease. Pneumonol Alergol Pol 2009, 77(3):305-313.

40. Martinez FJ, Han MK, Andrei AC, Wise R, Murray S, Curtis JL, Sternberg A, Criner G, Gay SE, Reilly J, Make B, Ries AL, Sciurba F, Weinmann G,
Mosenifar Z, DeCamp M, Fishman AP, Celli BR: Longitudinal change in the BODE index predicts mortality in severe emphysema. Am J Respir Crit Care Med 2008, 178(5):491-499.

41. Ong KC, Earnest A, Lu SJ: A multidimensional grading system (BODE index) as predictor of hospitalization for COPD. Chest 2005, 128(6):3810-3816.

42. Howell S, Coory M, Martin J, Duckett S: Using routine inpatient data to identify patients at risk of hospital readmission. BMC Health Serv Res 2009, 9:96.

43. Celli BR, Cote CG, Marin JM, Casanova C, Montes de Oca M, Mendez RA, Pinto Plata V, Cabral HJ: The Body-Mass Index Airflow Obstruction Dyspnea and Exercise Capacity Index in Chronic Obstructive Pulmonary Disease. N Engl J Med 2004, 350(10):1005-1012.

44. Esteban C, Quintana JM, Aburto M, Moraza J, Capelastegui A: A simple score for assessing stable chronic obstructive pulmonary disease. QJM 2006, 99(11):751-759.

45. American Thoracic Society: Standards for the diagnosis and care of patients with chronic obstructive pulmonary disease. Am J Respir Crit Care Med 1995, 152:S77-S120.

46. American Thoracic Society: Lung function testing: selection of reference values and interpretative strategies. Am Rev Respir Dis 1991, 144:1202-1218.

47. Omachi TA, Yelin EH, Katz PP, Blanc PD, Eisner MD: The COPD severity score: a dynamic prediction tool for health-care utilization. COPD 2008, 5(6):339-346.

48. Briggs A, Spencer M, Wang H, Mannino D, Sin DD: Development and validation of a prognostic index for health outcomes in chronic obstructive pulmonary disease. Arch Intern Med 2008, 168(1):71-79.

49. Kostianev SS, Hodgev VA, lluchev DH: Multidimensional system for assessment of COPD patients Comparison with BODE index. Folia Med (Plovdiv) 2008, 50(4):29-38.

50. Mehrotra N, Freire AX, Bauer DC, Harris TB, Newman AB, Kritchevsky SB, Meibohm B: Predictors of mortality in elderly subjects with obstructive airway disease: the PILE score. Ann Epidemiol 2010, 20(3):223-232.

51. Soler-Cataluna JJ, Martinez-Garcia MA, Sanchez LS, Tordera MP, Sanchez PR: Severe exacerbations and BODE index: two independent risk factors for death in male COPD patients. Respir Med 2009, 103(5):692-699.

52. Jones RC, Donaldson GC, Chavannes NH, Kida K, Dickson-Spillmann M, Harding S, Wedzicha JA, Price D, Hyland ME: Derivation and Validation of a Composite Index of Severity in Chronic Obstructive Pulmonary Disease - The DOSE Index. Am J Respir Crit Care Med 2009, 180(12):1189-95.

53. Niewoehner DE, Lokhnygina $Y$, Rice $K$, Kuschner W, Sharafkhaneh A, Sarosi GA, Krumpe P, Pieper K, Kesten S: Risk indexes for exacerbations and hospitalizations due to COPD. Chest 2007, 131:20-28.

54. Azarisman MS, Fauzi MA, Faizal MP, Azami Z, Roslina AM, Roslan H: The SAFE (SGRQ score air-flow limitation and exercise tolerance) Index: a new composite score for the stratification of severity in chronic obstructive pulmonary disease. Postgrad Med J 2007, 83(981):492-497.

55. Cardoso F, Tufanin AT, Colucci M, Nascimento O, Jardim JR: Replacement of the 6-min walk test with maximal oxygen consumption in the BODE Index applied to patients with COPD: an equivalency study. Chest 2007 132(2):477-482

56. Schembri S, Anderson W, Morant S, Winter J, Thompson P, Pettitt D, MacDonald TM, Winter $\mathrm{JH}$ : A predictive model of hospitalisation and death from chronic obstructive pulmonary disease. Respir Med 2009, 103(10):1461-1467.

57. Gruffydd-Jones K, Richman J, Jones RC, Wang X: A pilot study of identification and case management of high-risk COPD patients in a general practice. Fam Pract 2010, 27(5):494-8.

58. Graham I, Atar D, Borch-Johnsen K, Boysen G, Burell G, Cifkova R, Dallongeville J, de Backer G, Ebrahim S, Gjelsvik B, Herrmann-Lingen C, Hoes A, Humphries S, Knapton M, Perk J, Priori SG, Pyorala K, Reiner Z, Ruilope L, Sans-Menendez S, Op Reimer WS, Weissberg P, Wood D, Yarnell J, Zamorano JL, Walma E, Fitzgerald T, Cooney MT, Dudina A, Vahanian $A$, et al: European guidelines on cardiovascular disease prevention in clinical practice: full text Fourth Joint Task Force of the European Society of Cardiology and other societies on cardiovascular disease prevention in clinical practice (constituted by representatives of nine societies and by invited experts). Eur J Cardiovasc Prev Rehabil 2007, 14(Suppl 2):S1-113. 
59. van den Bemt L, Smeele I, van Weel C: Prognostic assessment of patients with COPD. Lancet 2009, 374(9705):1886-1887.

60. Wildman MJ, Sanderson C, Groves J, Reeves BC, Ayres J, Harrison D,

Young D, Rowan K: Implications of prognostic pessimism in patients with chronic obstructive pulmonary disease (COPD) or asthma admitted to intensive care in the UK within the COPD and asthma outcome study (CAOS): multicentre observational cohort study. BMJ 2007, 335(7630):1132.

61. Siebeling $L$, ter Riet $G$, van der Wal WM, Geskus RB, Zoller M Muggensturm P, Joleska I, Puhan MA: ICE COLD ERIC-International collaborative effort on chronic obstructive lung disease: exacerbation risk index cohorts-study protocol for an international COPD cohort study. BMC Pulm Med 2009, 9:15.

62. Vestbo J, Anderson W, Coxson HO, Crim C, Dawber F, Edwards L, Hagan G, Knobil K, Lomas DA, MacNee W, Silverman EK, Tal-Singer R: Evaluation of COPD Longitudinally to Identify Predictive Surrogate End-points (ECLIPSE). Eur Respir J 2008, 31(4):869-873.

63. Esteban C, Quintana JM, Moraza J, Aburto M, Aguirre U, Aguirregomoscorta Jl, Aizpiri S, Basualdo LV, Capelastegui A: BODE-Index vs HADO-score in chronic obstructive pulmonary disease: Which one to use in general practice? BMC Med 2010, 8:28.

64. Cote CG, Celli BR: Pulmonary rehabilitation and the BODE index in COPD. Eur Respir J 2005, 26(4):630-636

65. Nasis IG, Vogiatzis I, Stratakos G, Athanasopoulos D, Koutsoukou A, Daskalakis A, Spetsioti S, Evangelodimou A, Roussos C, Zakynthinos S: Effects of interval-load versus constant-load training on the BODE index in COPD patients. Respir Med 2009, 103(9):1392-1398.

66. Weston AD, Hood L: Systems biology proteomics and the future of health care: toward predictive preventative and personalized medicine. $J$ Proteome Res 2004, 3(2):179-196.

67. Wildman MJ, Sanderson C, Groves J, Reeves BC, Ayres J, Harrison D, Young D, Rowan K: Predicting mortality for patients with exacerbations of COPD and Asthma in the COPD and Asthma Outcome Study (CAOS). QJM 2009, 102(6):389-399.

doi:10.1186/1465-9921-12-151

Cite this article as: van Dijk et al:: Multidimensional prognostic indices for use in COPD patient care. A systematic review. Respiratory Research 2011 12:151.

\section{Submit your next manuscript to BioMed Central and take full advantage of:}

- Convenient online submission

- Thorough peer review

- No space constraints or color figure charges

- Immediate publication on acceptance

- Inclusion in PubMed, CAS, Scopus and Google Scholar

- Research which is freely available for redistribution

Submit your manuscript at www.biomedcentral.com/submit 